\title{
छs \\ Stable, tunable, quasimonoenergetic electron beams produced in a laser wakefield near the threshold for self-injection
}

\author{
S. Banerjee, ${ }^{*}$ S. Y. Kalmykov, N. D. Powers, G. Golovin, V. Ramanathan, ${ }^{\dagger}$ N. J. Cunningham, ${ }^{\ddagger}$ K. J. Brown, S. Chen, \\ I. Ghebregziabher, B. A. Shadwick, and D. P. Umstadter \\ Department of Physics and Astronomy, University of Nebraska, Lincoln, Nebraska 68588-0299, USA
}

B. M. Cowan and D. L. Bruhwiler ${ }^{\S}$

Tech-X Corporation, 5621 Arapahoe Avenue, Suite A, Boulder, Colorado 80303, USA

A. Beck" ${ }^{\|}$and E. Lefebvre

CEA, DAM, DIF, 91297 Arpajon Cedex, France

(Received 1 November 2012; published 25 March 2013)

\begin{abstract}
Stable operation of a laser-plasma accelerator near the threshold for electron self-injection in the blowout regime has been demonstrated with $25-60 \mathrm{TW}, 30$ fs laser pulses focused into a 3-4 millimeter length gas jet. Nearly Gaussian shape and high nanosecond contrast of the focused pulse appear to be critically important for controllable, tunable generation of $250-430 \mathrm{MeV}$ electron bunches with a lowenergy spread, $\sim 10 \mathrm{pC}$ charge, a few-mrad divergence and pointing stability, and a vanishingly small low-energy background. The physical nature of the near-threshold behavior is examined using threedimensional particle-in-cell simulations. Simulations indicate that properly locating the nonlinear focus of the laser pulse within the plasma suppresses continuous injection, thus reducing the low-energy tail of the electron beam.
\end{abstract}

DOI: $10.1103 /$ PhysRevSTAB.16.031302

PACS numbers: 52.38.Hb, 41.75.Jv, 52.38.Kd, 52.38.Ph

\section{INTRODUCTION}

Progress in the technology of short-pulse laser amplification has made it possible for laser-plasma accelerators (LPAs) to generate quasimonoenergetic (QME) electron beams [1-16] and to approach the GeV energy range [14-21]. Experiments show a direct correlation [22-24] between the generation of collimated electron beams and formation of a unique plasma structure-an electron density "bubble" - trailing the relativistically intense laser pulse [25-28]. The bubble forms behind the driver when the laser ponderomotive force creates complete electron cavitation (due to their high inertia, fully stripped ions remain immobile). Nonlinear evolution of the driver causes variations in the bubble shape, which triggers injection of ambient plasma electrons resulting in the formation of a

\footnotetext{
*Corresponding author. sudeep@unl.edu

Present address: Rensselaer Polytechnic Institute, Troy, New York 12180-3590, USA.

${ }^{\ddagger}$ Present address: Nebraska Wesleyan University, Lincoln, Nebraska 68504-2794, USA.

${ }^{\S}$ Present address: University of Colorado at Boulder, 390 UCB, Boulder, Colorado 80309-0390, USA.

"Present address: UMR 7638, Laboratoire Leprince-Ringuet, Ecole polytechnique, 91128 Palaiseau Cedex, France.
}

Published by the American Physical Society under the terms of the Creative Commons Attribution 3.0 License. Further distribution of this work must maintain attribution to the author(s) and the published article's title, journal citation, and DOI. collimated electron beam [29-33]. In order to meet the stringent requirements of applications, such as laserplasma based x-ray sources [34-39], LPAs must produce $\mathrm{GeV}$-scale energy, QME electron beams without a largedivergence, low energy, polychromatic background. Suppressing the background is important to minimize the noise produced in interactions, and improve the quality of secondary x-ray radiation [39]. Poorly collimated background electrons also represent a significant radiation hazard when an LPA operates at high repetition rates. The additional shielding required offsets one of the important advantages associated with LPAs.

Reduction of the electron energy spread and elimination of the low-energy spectral tails naturally occur when electrons are self-injected during a very short time interval, much shorter than the laser pulse transit time through the plasma. To generate background-free electron beams various schemes of externally controlled, localized injection have been proposed and realized in the laboratory [40]. However, only in a few of these experiments (viz. those using tapered-density gas targets) was stable production of such beams observed $[10,13]$. Theory and simulations suggest that controlling the evolution of the driving laser pulse can improve localization of electron injection, leading to the near elimination of the low-energy background $[31,33,41,42]$. Alternatively, matching the pulse for self-guiding (in a uniform plasma) and working close to the self-injection threshold can reduce backgrounds significantly $[28,43]$. Realization of this approach in the laboratory, however, appears to require very demanding 
laser pulse and target quality. Earlier experiments in the near-threshold regime with $100 \mathrm{TW}$-class lasers yielded polychromatic electron energy distributions, sometimes with a weak high-energy QME feature [15,18-20]. The polychromatic electron spectrum can be attributed to distortions in the incident laser pulse, resulting in reduced coupling of laser energy to the accelerating structure, leading to large fluctuations of the charge and energy. Even with an ideal laser pulse, an optical shock can form long before electron dephasing and cause continual elongation of the bubble leading to continuous electron injection [32,33]. For example, a 50-60 fs, 60-200 TW laser pulse, propagating through the plasma with the electron density $n_{e} \sim 5 \times 10^{18} \mathrm{~cm}^{-3}[15,18-20]$, rapidly evolves into a relativistic optical shock with a very steep front [44,45]. In this situation, a massive polychromatic tail would develop even if self-injection during the early stages of the interaction produces a QME bunch $[18,19]$.

In this paper, we demonstrate that the near-threshold regime can be accessed in a stable manner, yielding high electron-beam quality over a broad range of laser and plasma parameters. Simulations indicate that (for the experiments discussed here) the threshold for self-injection is determined by the location of the nonlinear laser focus relative to the exit of the plasma [16] rather than by the power threshold for relativistic self-focusing $[19,20]$. The nearly Gaussian radial profile of the focused 25-60 TW laser beam and stable operation of the laser system allowed for maintaining the favorable focusing geometry over long series of shots, precisely locating the nonlinear focus in the 3 to $4 \mathrm{~mm}$ length plasma. Thus, location and duration of self-injection was well controlled, yielding tunable production of 130-430 MeV electron beams with narrow energy spread and minimal low-energy background. For some parameters, over long series of shots, electron beams were produced with the complete absence of low-energy background. The tunability of the beam energy and stability of the acceleration process demonstrated here are vital for radiation-physics applications [34-39].

The paper is organized as follows. Section II describes the experimental setup. Section III discusses the stability of the quasimonoenergetic acceleration process over a broad range of plasma densities and laser pulse energies. Section IV provides a theoretical interpretation of the observations based on three-dimensional particle-in-cell (PIC) simulations. Section V summarizes the results.

\section{EXPERIMENTAL SETUP}

The experiments were carried out with the $100 \mathrm{TW}$ Diocles laser [16,34]. This system produces linearly polarized pulses with a central wavelength $\lambda_{0}=0.805 \mu \mathrm{m}$, pulse duration (full width at half maximum, or FWHM, in intensity) $\tau_{L}=30 \mathrm{fs}$, and maximum energy of $3.5 \mathrm{~J}$ at a $10 \mathrm{~Hz}$ repetition rate. The laser beam is incident on a deformable mirror that operates in a feedback loop with a wave front sensor. The $70 \mathrm{~mm}$-diameter laser pulse, with spatial aberrations corrected, is focused to a nearly diffraction limited spot (Strehl ratio $\sim 0.95$ ) using a one meter focal length, dielectric-coated off-axis paraboloid. The intensity profile in the central spot (shown in the inset of Fig. 1) allows a Gaussian fit with a radius $r_{0}=13.6 \mu \mathrm{m}$; $70 \%$ of the incident energy is contained within a diameter $2 r_{0}$. The results reported here were obtained with 25$60 \mathrm{TW}$ on target. The laser pulse was spatially and temporally characterized at full power using a multistage beam sampling system. The energy on target was varied using a combination of a wave plate and polarizers. The temporal duration was optimized by varying the separation between the compressor gratings, and adjusting the second and third order phase by use of a spectral phase modulator. The shot-to-shot pointing stability of the laser pulse on target was $\pm 7 \mu \mathrm{rad}$.

The experimental setup is presented in Fig. 1. The target was a high-density jet of neutral helium produced by a 3 or 4 mm-diameter supersonic Laval nozzle; most results reported below (except those shown in Fig. 9) were obtained with the $3 \mathrm{~mm}$ nozzle. The neutral density profile (characterized interferometrically) has half-millimeter-long symmetric down-ramps along the edges, and a 2 or $3 \mathrm{~mm}$ central flat region. The laser pulse was focused before the front edge of the nozzle. During the experiments, an equivalent plane imaging system locates the longitudinal position of the focal plane with a precision $\pm 0.5 \mathrm{~mm}$. The focused pulse fully ionizes the medium producing a plasma with $n_{e}=(0.4-5) \times 10^{19} \mathrm{~cm}^{-3}$. At $n_{e}<2 \times 10^{19} \mathrm{~cm}^{-3}$, images of the plasma emission at $800 \mathrm{~nm}$ show a uniform laser-created plasma column spanning the entire jet length. The accelerated electrons exiting the plasma impinge on a fluorescent screen (LANEX) that was imaged with a 12-bit

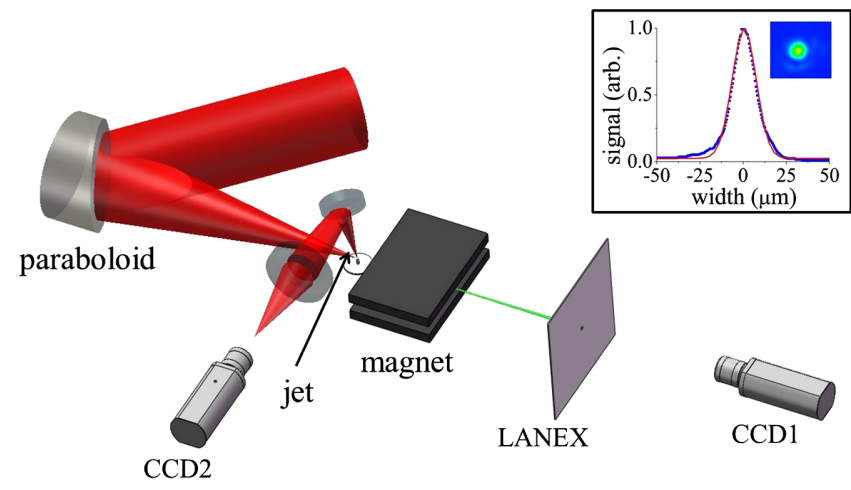

FIG. 1. Schematic of the experimental setup. The laser pulse is focused at the front edge of a supersonic helium gas jet. Pulse propagation is monitored by imaging the Thomson-scattered light using CCD2. Electrons passing through a magnetic spectrometer impinge on a LANEX screen. Optical emission from the LANEX is imaged onto CCD1 to measure the beam angular divergence and energy spectrum. Inset: image of the laser focal spot in vacuum (full-power shot) and its vertical (blue/dark gray) and horizontal (red/light gray) lineouts. 
CCD. The absolute response of LANEX to electrons, calibrated using an $18 \mathrm{MeV}$ radio-frequency linear accelerator (Siemens Primus), was used to obtain the charge in a specified energy interval. The sensitivity and dynamic range of the detection system depends on the dispersion and angular divergence. For the conditions reported in this work, the achievable dynamic range exceeds $10^{3}$ for beam divergence in the range of 3-5 mrad. Electron energy was measured using a magnetic spectrometer operating in a slit-free geometry and having a range of $20-500 \mathrm{MeV}$. The energy resolution was better than $10 \%$ at $300 \mathrm{MeV}$, and rapidly degraded beyond $400 \mathrm{MeV}$. The spectrometer response function has been modeled with the General Particle Tracer (GPT) code [46,47]. The final energy distribution was obtained taking into account the finite beam divergence.

\section{PRODUCTION OF LOW-ENERGY SPREAD ELECTRON BEAMS}

With the laser power and target length fixed, the plasma density was scanned by varying the backing pressure on the nozzle until electron beams of the highest quality were produced. The position of the laser focal plane with respect to the nozzle was kept constant over the entire series of shots. Below, we provide a detailed analysis of two representative series of shots carried out with a $3 \mathrm{~mm}$ nozzle and 26 and 43 TW on target, demonstrating the features of the near-threshold regime in high- and low-density plasmas. In both cases, electron beams produced at densities above $2.5 \times 10^{19} \mathrm{~cm}^{-3}$ had large divergence, broad energy spectra, and large shot-to-shot variation of parameters. Monoenergetic features first emerged at $n_{e} \approx 2.5 \times$ $10^{19} \mathrm{~cm}^{-3}$. A further decrease in the density caused a reduction of the energy spread and charge, with a corresponding increase in peak energy and suppression of the low-energy tail.

\section{A. Pointing stability and beam collimation}

The pointing stability of the beam and the divergence of the entire ensemble of accelerated electrons were evaluated by allowing the electrons exiting the plasma to strike the LANEX without magnetic deflection. The sample shots, corresponding to the low-background, QME beams obtained with 26 and $43 \mathrm{TW}$ (at densities $n_{e}=1.2 \times 10^{19}$ and $7.2 \times 10^{18} \mathrm{~cm}^{-3}$, respectively), are shown in Figs. 2(a)-2(f). Comparison of these sample shots demonstrate that, at lower power/higher density, the divergence of the entire beam is consistently higher than in the lowdensity case, $6 \mathrm{mrad}$ vs $3 \mathrm{mrad}$. However, as we shall see in the following subsection, the main contributor to the halo in the high-density case is the remaining low-energy background, with the QME component having $4 \mathrm{mrad}$ divergence. The slight asymmetry of the beam in the high-density case may be attributed to the direct interaction

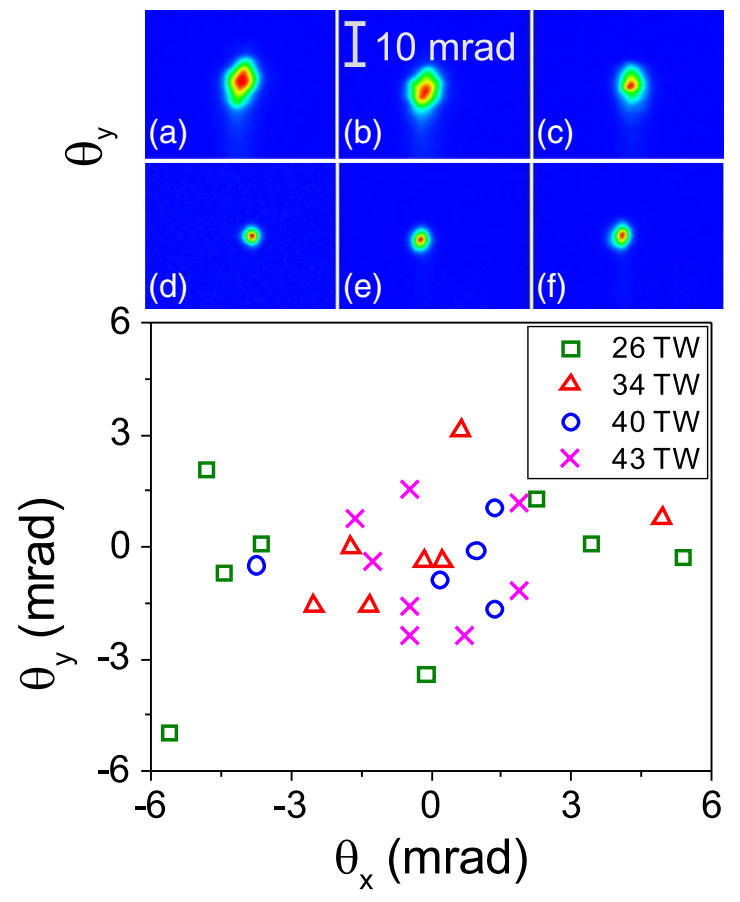

FIG. 2. Images of electron beam on LANEX without dispersion in magnetic spectrometer; the color scale is normalized for each shot. (a)-(c) Electron beams obtained with 26 TW laser power and plasma density $1.2 \times 10^{19} \mathrm{~cm}^{-3}$. The beam divergence is $6 \mathrm{mrad}$ (FWHM). (d)-(f) Electron beams obtained with $43 \mathrm{TW}$ laser power and plasma density $7.2 \times 10^{18} \mathrm{~cm}^{-3}$. The beam divergence is $3 \mathrm{mrad}$ (FWHM). The plot at the bottom shows the angular position of the undeflected beam (calculated from the centroid) for a laser power in the range between 26 and $43 \mathrm{TW}$, and plasma density in the range $6 \times 10^{18}-2.4 \times$ $10^{19} \mathrm{~cm}^{-3}$. Symbols correspond to the following values of laser power and electron density: $(\square) 26 \mathrm{TW}, 9 \times 10^{18}-2.4 \times$ $10^{19} \mathrm{~cm}^{-3},(\triangle) 34 \mathrm{TW}, 8 \times 10^{18}-1.2 \times 10^{19} \mathrm{~cm}^{-3}$, (○) $40 \mathrm{TW}$, $7.2 \times 10^{18}-1.2 \times 10^{19} \mathrm{~cm}^{-3}$, and $(\times) \quad 43 \mathrm{TW}, 6 \times 10^{18}-9 \times$ $10^{18} \mathrm{~cm}^{-3}$. Over the entire range of variation of the laser and plasma parameters, lateral deflection of the beam (the beam pointing) does not exceed $4 \mathrm{mrad}$.

of injected particles with the linearly polarized laser radiation filling in the bubble [5].

As can be seen from the bottom panel of Fig. 2, in the entire range of laser power (from 26 to $43 \mathrm{TW}$ ) and electron density (from $n_{e}=6 \times 10^{18}$ to $2.4 \times 10^{19} \mathrm{~cm}^{-3}$ ), the lateral deflection of the beam centroid is less than $4 \mathrm{mrad}$. The dispersion of the beam pointing does not exceed the beam divergence, in striking contrast to the case of QME electron acceleration in very dense plasmas, $n_{e} \sim 5 \times 10^{19} \mathrm{~cm}^{-3}$ (with sub-30 TW laser pulses) $[11,12]$. In these high-density experiments, the collimated components of electron bunches had roughly $3 \mathrm{mrad}$ divergence, with the pointing dispersion nearly an order of- magnitude higher [11]. Moreover, the divergence of the halo surrounding the collimated component was nearly a factor 5 greater than the entire beam divergence in 
Figs. 2(a)-2(c). This comparison clearly shows benefits of using low-density plasmas as accelerating structures. A low risk of laser filamentation and larger size of the bubble (reducing the interaction of electron bunch with the laser pulse tail) indeed improve the beam pointing stability and collimation.

In agreement with the earlier studies [48], the pointing stability of the beams (as well as other parameters) are found to be sensitive to the pulse contrast. For the nanosecond contrast $>5 \times 10^{-8}$, beam parameters strongly fluctuated from shot to shot. The results reported here correspond to a nanosecond contrast of $2 \times 10^{-8}$, achieved by optimizing the timing of the Pockel cells in the laser chain, and the time delay between the pump and the seed beams in all amplification stages. This optimization resulted in the generation of stable beams in a broad range of laser and plasma parameters; examples of the stable accelerator performance are presented in Sec. III C.

\section{B. Quasimonoenergetic electron beams from high-density plasmas}

The lowest laser power at which QME electron beams could be reliably obtained with the $3 \mathrm{~mm}$ jet was found to be $26 \mathrm{TW}$. At this power, no accelerated electrons were detected below $n_{e}=9 \times 10^{18} \mathrm{~cm}^{-3}$.

LANEX images of magnetically dispersed electron beams in Figs. 3(h), 3(g), and 3(f) show the progress

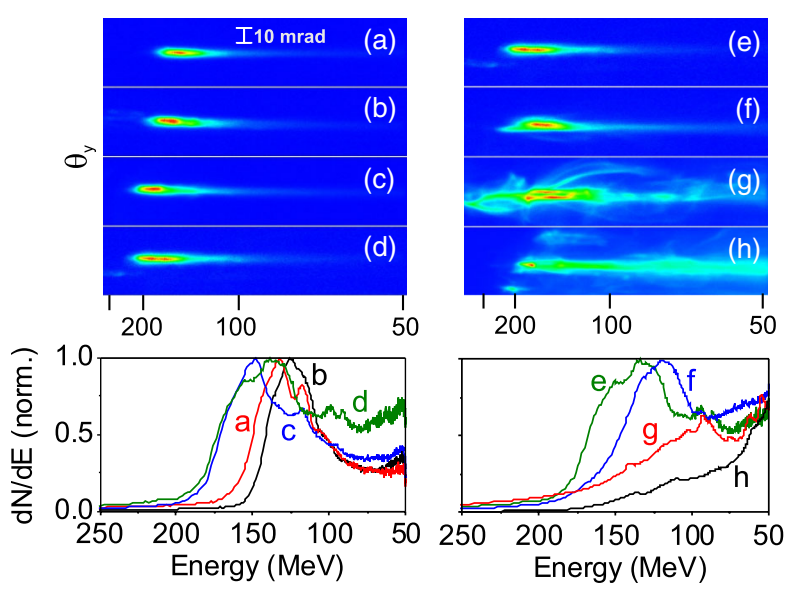

FIG. 3. Electron energy spectra obtained for laser power of $26 \mathrm{TW}$; the color scale is normalized for each shot. The left column [panels (a)-(d) and the plot at the bottom] shows LANEX images and corresponding lineouts for the lowest plasma density $\left(1.2 \times 10^{19} \mathrm{~cm}^{-3}\right)$, yielding stable, QME electron beams. The energy corresponding to the spectral peak fluctuates between 130 and $160 \mathrm{MeV}$. The integrated charge in this energy interval is $18 \pm 2.3 \mathrm{pC}$ and the beam divergence is $4 \mathrm{mrad}$. The right column [panels (e)-(h) and the plot at the bottom] shows that the electron spectra evolve from QME to quasithermal distributions as the plasma density increases: (e) 1.2 , (f) 1.5 , (g) 1.8 , and (h) $2.4 \times 10^{19} \mathrm{~cm}^{-3}$. Panels (d) and (e) represent the same laser shot. from a broad, thermal-like electron energy distribution towards the spectrum with the QME peak at approximately $120 \mathrm{MeV}$. This transition, occurring as the density drops from $2.4 \times 10^{19}$ to $1.8 \times 10^{19}$ to $1.5 \times 10^{19} \mathrm{~cm}^{-3}$, can be partly understood as a transition from the "selfmodulated" regime with injection and acceleration occurring in several subsequent buckets [49] to the standard blowout regime with injection and acceleration in a single bucket $[5,27]$ immediately trailing the laser pulse. Indeed, when the electron density exceeds $1.5 \times 10^{19} \mathrm{~cm}^{-3}$, the laser pulse length $\left(\tau_{L}=30 \mathrm{fs}\right)$ is longer than the electron plasma period, $\tau_{p}=2 \pi / \omega_{p e}$, and the laser power, $26 \mathrm{TW}$, exceeds the critical power for the relativistic self-focusing, $P_{\text {cr }}=16.2\left(\omega_{0} / \omega_{p e}\right)^{2} \mathrm{GW}[50]$, by more than a factor 13 . (Here, $\omega_{0}=2 \pi c / \lambda_{0}$ is the laser carrier frequency, $\omega_{p e}=$ $\sqrt{4 \pi e^{2} n_{e} / m_{e}}$ is the electron Langmuir frequency, $m_{e}$ is the electron rest mass, $-|e|$ is the electron charge, and $c$ is the speed of light in vacuum.) This situation is potentially unstable, leading to the catastrophic self-focusing [26], longitudinal breakup, and hosing of the laser beam [49]. In addition, for $n_{e}>1.5 \times 10^{19} \mathrm{~cm}^{-3}$, the pulse depletion length, $L_{d} \approx\left(\omega_{0} / \omega_{p e}\right)^{2} c \tau_{L}$ [28], is less than $0.8 \mathrm{~mm}$ (i.e., less than $40 \%$ of the density plateau). As a result, the pulse almost instantly develops a very sharp leading edge (relativistic optical shock) [44], causing bubble distortions and continuous injection $[18,32,33]$. In these circumstances, production of QME beams is not to be expected. In addition, the relativistically intense laser radiation filling the bubble interferes with the electron betatron oscillations $[5,38,51]$. As a result, the electron beams detected at $n_{e}=$ $1.8 \times 10^{19}$ and $2.4 \times 10^{19} \mathrm{~cm}^{-3}$ [cf. Figs. 3(g) and 3(h)] develop quite a complicated transverse structure (also observed in similar circumstances in other experiments $[23,38])$.

For plasma density below $1.5 \times 10^{19} \mathrm{~cm}^{-3}$ [cf. Figs. 3(f) and 3(e)], the laser pulse becomes almost entirely confined within the bubble, improving the stability of the system. The energy distributions, however, still show significant low-energy background [cf. curves (a)-(f) in the bottom panels of Fig. 3], which resembles the spectra observed by others under similar conditions [7]. The presence of the energy tails may be explained by incomplete suppression of the continuous injection; the optical shock still forms before the end of the plasma, driving bubble expansion and injection. The speed of shock formation (also known as the "pulse front etching") is determined by factors including group velocity dispersion in the plasma [44,52]. Slower etching at lower plasma densities results in slower expansion of the bubble, reducing injected charge [cf. the signal below $100 \mathrm{MeV}$ in Figs. 3(a)-3(c)].

Figures 3(a)-3(e) show LANEX images of the most stable QME electron beams obtained at $n_{e}=1.2 \times$ $10^{19} \mathrm{~cm}^{-3}$. At this density, the accelerated electrons rapidly outrun the bubble and enter the decelerating phase, the dephasing length $[28,53]$ being $L_{\mathrm{deph}} \approx 0.6 \mathrm{~mm}$. As the 
earlier injected electrons slow down, electrons injected later keep accelerating, and the energy peak forms at the high-energy end of a relatively flat distribution [54]. The central energy of the peak (varying from shot to shot between 130 and $160 \mathrm{MeV}$ ) is lower than the $220 \mathrm{MeV}$ predicted by the scaling laws [28]. This is partly due to beam loading [55], but mostly because the laser pulse depletes long before the end of the plasma, leaving the electron bunch to drive its own wake, losing energy in the process (cf. the scenario discussed in Ref. [22]). This may also explain the relatively large, $30 \%-40 \%$, energy spread. The electrons making up the energy peak have $4 \mathrm{mrad}$ divergence, which is lower than the $6 \mathrm{mrad}$ divergence of the entire undispersed beam [cf. Figs. 2(a)-2(c)]. The halo (produced by the low-energy electrons) appears to be fairly weak, in contrast to the experiments with high-density plasmas [11].

No accelerated electrons were detected for $n_{e}<9 \times$ $10^{18} \mathrm{~cm}^{-3}$. This termination of injection cannot be explained by the insufficient power for self-focusing, as $P / P_{\mathrm{cr}} \approx 8$, and $\omega_{p e} \tau_{L} \approx 5$. Given nearly the Gaussian transverse profile of the incident pulse, the pulse energy coupling to the structure is expected to be high. However, if nonlinear pulse focusing and formation of the bubble are delayed (which may occur, for instance, if the pulse is focused at a $\sim 1 \mathrm{~mm}$ distance before the edge of the nozzle), the plasma may be too short for the self-injection to occur [16]. This physical scenario of termination of selfinjection is discussed in detail in Sec. IV.

\section{Background-free quasimonoenergetic electron beams from low-density plasmas}

\section{Self-injection threshold at low density}

Reduction of the plasma density increases the dephasing length, reduces the rate of pulse depletion, and mitigates undesirable nonlinear optical phenomena (such as optical shock formation), thus increasing both electron-beam energy and quality. Figure 4 shows LANEX images of magnetically dispersed beams and corresponding lineouts for representative shots taken with the $43 \mathrm{TW}$ pulse at values of the plasma density between $6 \times 10^{18}$ and $9.6 \times$ $10^{18} \mathrm{~cm}^{-3}$. Over the entire range of densities, the electron energy spectra show either weak or no background, with the QME component being the dominant spectral feature. The peak of the QME distribution is $320 \mathrm{MeV}$ for the lowest density of this sequence and decreases to $130 \mathrm{MeV}$ for the highest density.

When the density is high, $9.6 \times 10^{18}$ and $8.4 \times$ $10^{18} \mathrm{~cm}^{-3}$, the QME peak is accompanied by a fairly bright tail extending to the cutoff of the spectrometer; this is similar to the spectra presented in Figs. 3(a)-3(e). The total charge (calculated from the entire LANEX image plate) increases as the plasma density increases, partly due to the contribution from the tail. This trend has the same origin as in the high-density case discussed in the
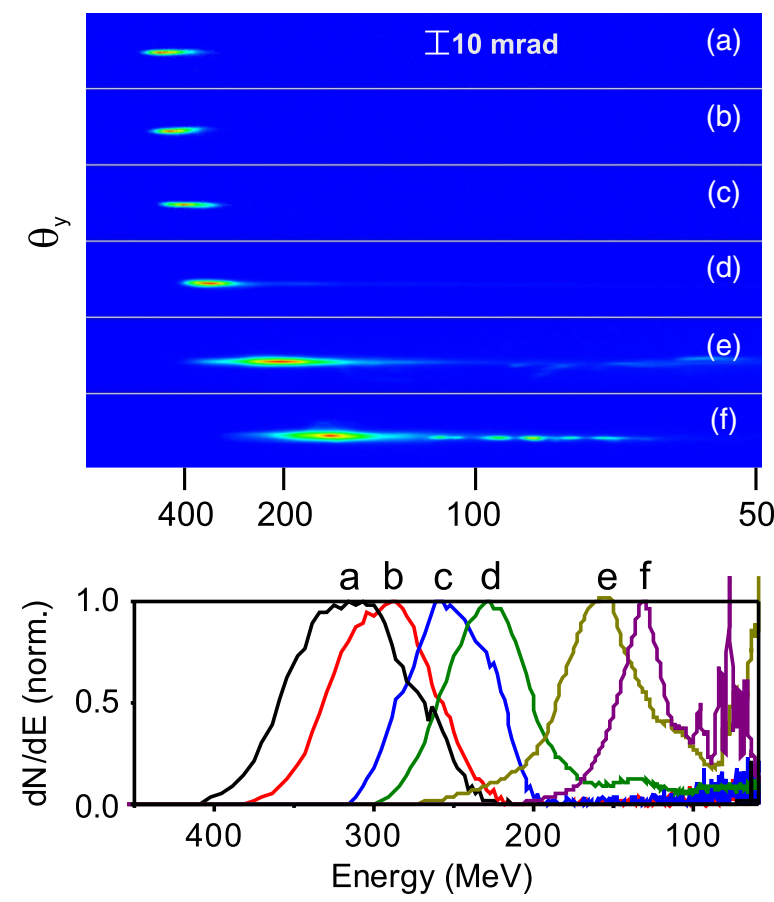

FIG. 4. QME electron spectra as a function of plasma density for the laser power of $43 \mathrm{TW}$. The values of the plasma density are (a) 6, (b) 6.6, (c) 7.2, (d) 7.8, (e) 8.4 , and (f) $9.6 \times 10^{18} \mathrm{~cm}^{-3}$. The corresponding charge on the LANEX screen is (a) 3 , (b) 3 , (c) 7, (d) 15, (e) 32, and (f) $45 \mathrm{pC}$. The angular divergence of the beam measured along the nondispersed axis is $\sim 3 \mathrm{mrad}$ for the panels (a)-(d) and $\sim 4$ mrad for (e)-(f). The color scale is normalized for each shot.

Sec. III B. Violent nonlinear evolution of the laser pulse leads to higher injected charge and continuous injection, with electrons reaching dephasing long before the end of the plasma. Rapid dephasing limits the energy gain and produces the broad QME peak at the high-energy end of the distribution [cf. lineouts (e) and (f) in the bottom panel of Fig. 4]. Furthermore, rapid pulse depletion (the depletion length at $8.4 \times 10^{18} \mathrm{~cm}^{-3}$ being $1.1 \mathrm{~mm}$ ) results in the electron beam driving its own wake near the end of the plasma, losing energy, and accumulating a low-energy tail. As a result, the energy gain is almost 50\% lower than that predicted by standard scalings [28,53]. Remarkably, in spite of the strongly nonlinear character of the interaction, the beams in the high-density regime are well reproducible from shot to shot (cf. Fig. 5).

Decreasing the plasma density helps to reduce the tail and to increase the beam energy; nonetheless, a weak flux of electrons is observed below $175 \mathrm{MeV}$ in all shots at $n_{e}=$ $7.8 \times 10^{18} \mathrm{~cm}^{-3}$. A typical spectrum is lineout (d) in the bottom panel of Fig. 4. Below this density, the increase of the peak energy is accompanied by further reduction, and, eventually, by complete disappearance of the tail, as can be seen in the lineouts (a) and (b) in the bottom panel of Fig. 4. These lineouts show no measurable electron signal below $200 \mathrm{MeV}$ for the lowest densities of the sequence, $6 \times 10^{18}$ 

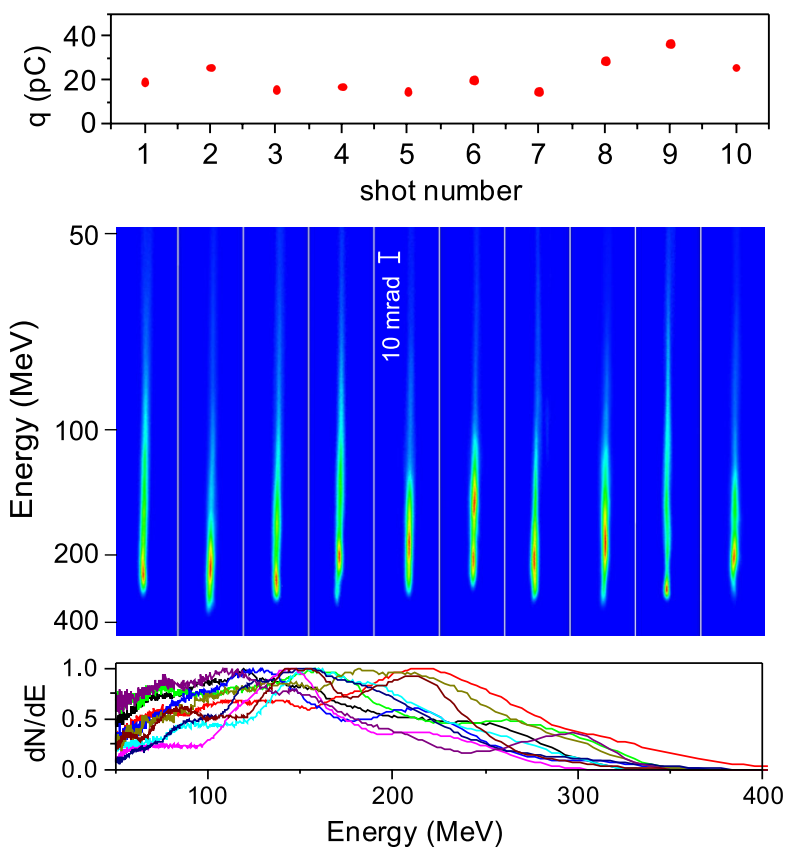

FIG. 5. LANEX images of spatially dispersed beams and spectral lineouts (bottom panel) for ten consecutive shots taken at $n_{e}=1.1 \times 10^{19} \mathrm{~cm}^{-3}$ and $P=41 \mathrm{TW}$. The color scale is normalized for each shot. The top panel shows the integrated charge accounting for background noise on the detector.

and $7.2 \times 10^{18} \mathrm{~cm}^{-3}$. Such beams are typically observed in roughly $50 \%$ of shots (cf. Fig. 6); particularly, the images in Figs. 4(a) and 4(b) were selected from the long sequences of background-free shots similar to the one presented in Fig. 7. These highest-energy, backgroundfree beams have just a few $\mathrm{pC}$ charge. Their remarkable shot-to-shot stability is demonstrated in Sec. IIIC2 (cf. Figs. 6-8). Electron beams are intermittently observed at $n_{e}=5.4 \times 10^{18} \mathrm{~cm}^{-3}$, and are not detected at all below this limit. The physical nature of this threshold is discussed in detail in Sec. IV.

Multishot electron-beam statistics for each parameter set of Fig. 4 are summarized in Table I. For each series of shots, the energy spread (FWHM of the QME feature) is on the order of $25 \%$. The low-energy background of the beam is quantified by computing the contrast, defined as the ratio of the charge within the FWHM of the QME peak to the total charge on the LANEX. Presence of the background significantly reduces the contrast. Indeed, the optimal beams [entries (a)-(c) of Table I] have the highest contrast, $C \approx 0.7$, close to that of the ideal Gaussian distribution. Thus, in these shots, the charge is concentrated in the QME peak. The low-energy tail emerging at higher densities consistently reduces the contrast down to $C \approx 0.4$ [entry (f) of Table I]. Remarkably, the pointing angle fluctuation is below the beam divergence in the entire range of densities, $6 \times 10^{18}-9.6 \times 10^{18} \mathrm{~cm}^{-3}$, which is the opposite of the observations made in earlier experiments with the high-density plasmas $[8,11,12]$. At the optimal
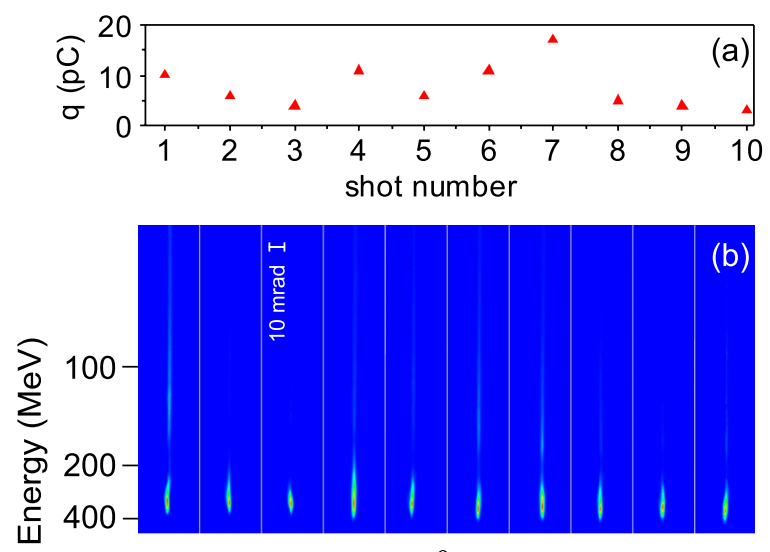

$\theta$
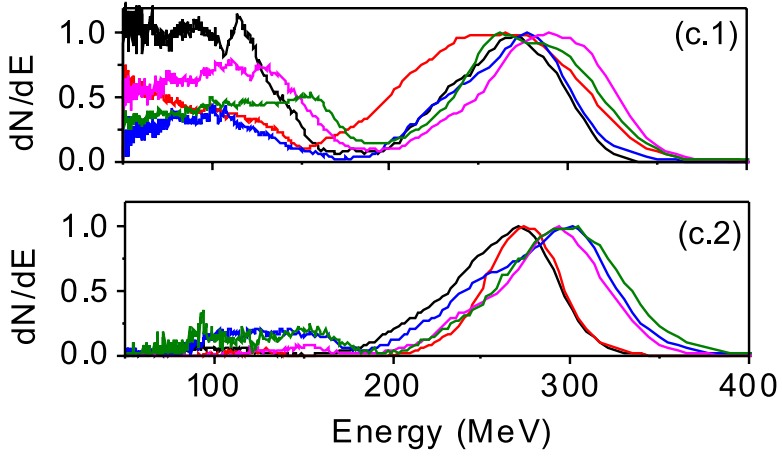

FIG. 6. LANEX images of magnetically dispersed beams [panel (b)] and corresponding spectral lineouts [panels (c.1) and (c.2)] for ten consecutive shots taken at $n_{e}=6 \times$ $10^{18} \mathrm{~cm}^{-3}$ and $P=43 \mathrm{TW}$; the color scale is normalized for each shot. Panel (a) shows the integrated charge, taking into account background noise on the detector. All beams have a QME feature with the energy $\approx 300 \mathrm{MeV}$, carrying a few $\mathrm{pC}$ charge. Shots no. 2, 3, and 8-10 show no significant electron signal below $200 \mathrm{MeV}$ [lineouts in panel (c.2)]. Presence of the background in other shots [lineouts in panel (c.1)] raises the charge to $10-20 \mathrm{pC}$.

operation point, $n_{e}=6 \times 10^{18} \mathrm{~cm}^{-3}$, the measured pointing fluctuation reaches a minimum of $\pm 1.1 \mathrm{mrad}$.

\section{Stability of the acceleration process}

Over the entire range of densities $6 \times 10^{18}-11 \times$ $10^{18} \mathrm{~cm}^{-3}$, generation of electron beams using a nearly ideal laser pulse of the power $>40 \mathrm{TW}$ is seen to be very stable. The key to this remarkable reproducibility is the stability of the laser system and gas-jet target. Thus, even though the laser-plasma interaction is highly nonlinear, the fluctuations of the initial parameters are minimized, reducing the consequences of the nonlinearities. This stability allows for operation near the self-injection threshold without fluctuation pushing the parameters too far from the threshold, having catastrophic effects on beam quality. To support this claim, we show two series of ten consecutive shots for $n_{e}=1.1 \times 10^{19} \mathrm{~cm}^{-3}, P=41 \mathrm{TW}$ (cf. Fig. 5) and $n_{e}=6 \times 10^{18} \mathrm{~cm}^{-3}, P=43 \mathrm{TW}$ (cf. Fig. 6). 


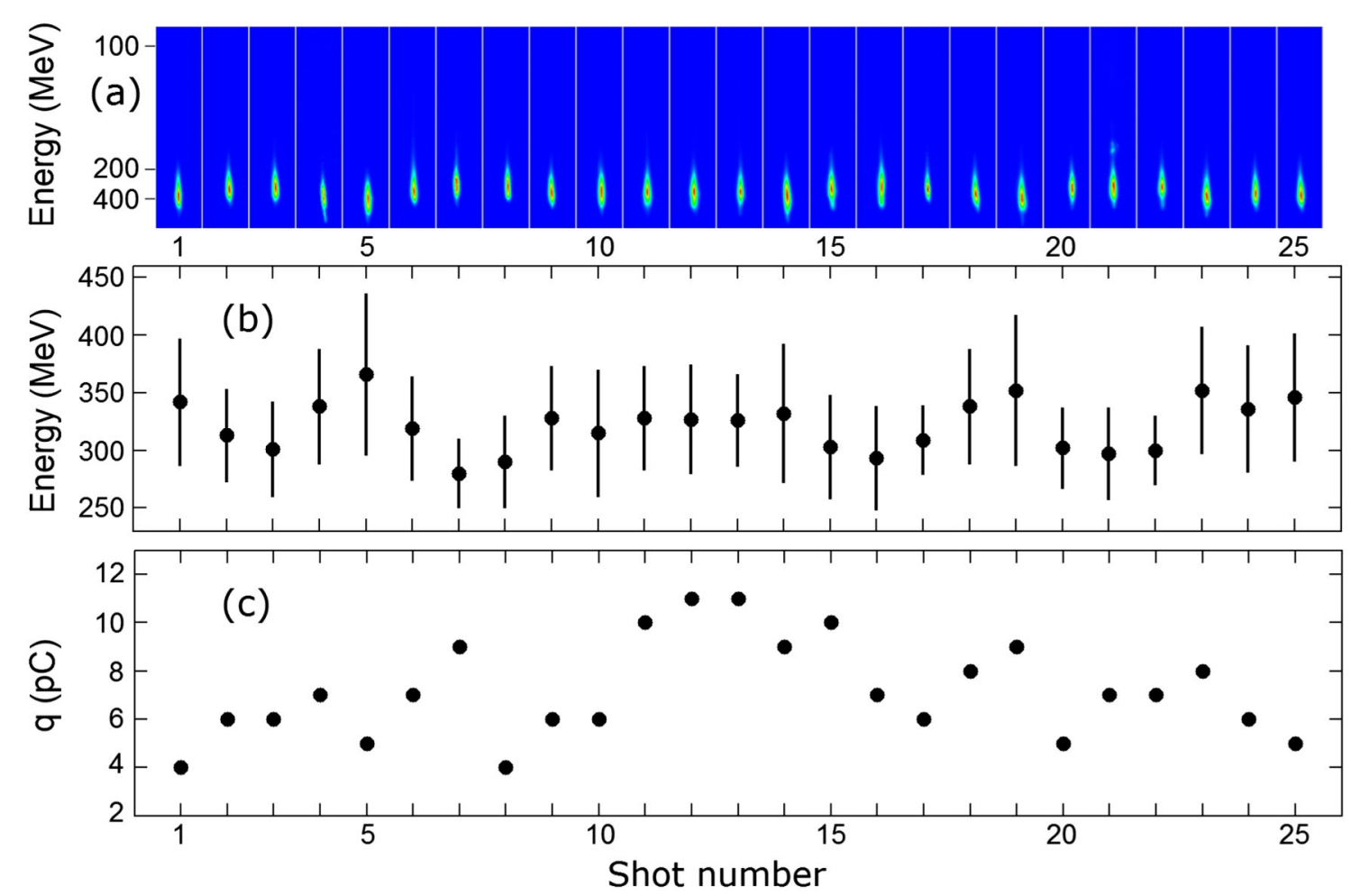

FIG. 7. (a) LANEX images of magnetically dispersed beams for 25 consecutive shots taken at $n_{e}=6 \times 10^{18} \mathrm{~cm}^{-3}$ and $P=$ $55 \mathrm{TW}$; the color scale is normalized for each shot. In all cases, the low-energy tail is not detected at all. The beam pointing fluctuation for this series of shots is $\pm 1 \mathrm{mrad}$. (b) Electron energy corresponding to the spectral peak (markers) and FWHM energy spread (bars) vs shot number. (c) Integrated charge, taking into account background noise on the detector.

The series of shots in Fig. 5 shows that electron beams can be consistently produced in a high-density plasma, though with very broad spectra and no pronounced QME features. The energy distribution is consistent with continuous injection of electrons into the plasma bubble, leading to the high charge in the beam. The series shown in Fig. 6, taken at approximately half the density of that of Fig. 5, demonstrates the stability of near-threshold operation. In Fig. 6 we see two types of beams, both with the QME feature centered around $E \approx 300 \mathrm{MeV}$. One has a negligible energy tail below $200 \mathrm{MeV}$ (shots no. 2, 3, 8-10), and the other has the tail comparable in flux to the QME feature. For both types of beams, the QME feature contains a few $\mathrm{pC}$ of charge; with the tail present, the charge raises to $10-20 \mathrm{pC}$. As one can see in Fig. 6, the electron beams with a negligible background show up in roughly $50 \%$ of the shots, provided the LPA works near threshold for self-injection.

One important benefit of the near-threshold regime is that the background-free beams are often observed in long series of shots; one representative series is shown in Figs. 7 and 8. Electron spectra shown in Fig. 8(a) have, on average, 29\% normalized FWHM. The FWHMs of individual spectra are shown as bars in Fig. 7(b). The spectral peak corresponds, on average, to the $321 \mathrm{MeV}$ energy, with a $22 \mathrm{MeV}$ standard deviation over the series of shots. This deviation is better than the experimental uncertainty brought about by the coarse spectrometer resolution $(\sim 10 \%$ for $E \approx 300 \mathrm{MeV})$. The mean integrated charge is $7.16 \mathrm{pC}$ with a standard deviation of $2.03 \mathrm{pC}$. Remarkably, within a resolution of the energy measurement, there is no evidence of correlation of electron energy with charge. This is consistent with the simulation data for similar regimes, showing that, to cause observable manifestation of beam loading (viz. more than $10 \%$ reduction of electron energy), the injected charge should be at least an order of magnitude higher [33].

\section{Tunability and scalability}

The near-threshold regime yielding stable low- and zerobackground beams is scalable: the optimal operating density can be found for different laser powers and target lengths. Examples of these optimal beams are presented in Fig. 9. These beams were obtained with a longer plasma, using a $4 \mathrm{~mm}$-diameter Laval nozzle and with the laser power of 40, 51, and $58 \mathrm{TW}$. For each value of the power, a density was found at which a background-free electron beams were produced, with spectral peaks at 276, 390, and $430 \mathrm{MeV}$, respectively. As the laser power increases, the optimal density for the QME beam generation reduces, and electron energy grows; the energy spread, however, increases as well. 

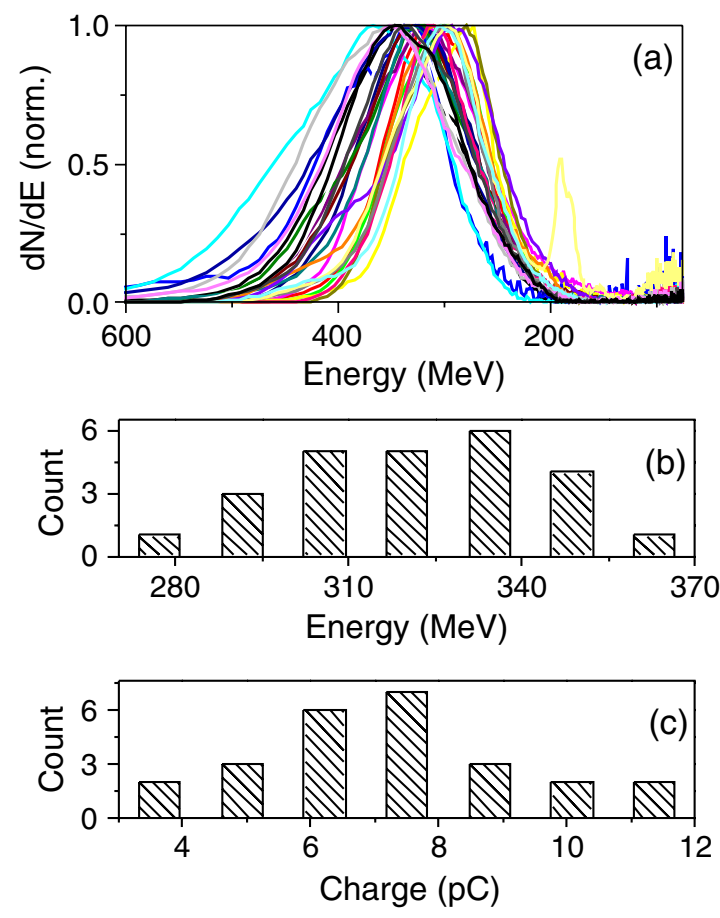

FIG. 8. (a) Energy spectra for individual shots, (b) statistics of energy corresponding to the spectral peak, and (c) statistics of charge over the series of 25 shots of Fig. 7. The energy histogram shows seven bins between 270 and $340 \mathrm{MeV}$ (bin size $\Delta E \approx$ $14 \mathrm{MeV})$. The charge histogram shows seven bins between 3 and $12 \mathrm{pC}(\Delta Q \approx 1.3 \mathrm{pC})$.

\section{Summary of experimental results}

The acceleration process in the near-threshold regime, leading to generation of low-background (in some cases, with no detectable background), QME electron beams over a long series of consecutive shots, is remarkably stable. Charge, pointing, and energy of the QME feature show minimal fluctuations and appear to be highly reproducible

TABLE I. Electron-beam characteristics for the parameters of Fig. 4 averaged over $10-20$ shots. $n_{e}$ is the electron density, $E$ is the central energy in $\mathrm{MeV}, \Delta E / E$ is the normalized energy spread (FWHM), $D$ is the FWHM divergence in mrad, $P$ is the pointing stability in mrad, $Q$ is the average integrated charge in $\mathrm{pC}, C$ is the contrast defined as the ratio of beam charge within FWHM in energy to the total beam charge, and $B$ is the brightness per shot in units of $10^{10}$ electrons $\mathrm{MeV}^{-1} \mathrm{~mm}^{-2} \mathrm{mrad}^{-2}$. In every series of shots, the measured fluctuation in the total charge is $\sim 50 \%$ (rms).

\begin{tabular}{ccccccccc}
\hline \hline & $n_{e}\left(\mathrm{~cm}^{-3}\right)$ & $E$ & $\Delta E / E$ & $D$ & $P$ & $Q$ & $C$ & $B$ \\
\hline (a) & $6.0 \times 10^{18}$ & 315 & 0.25 & 3.1 & \pm 1.7 & 3 & 0.7 & 4.4 \\
(b) & $6.6 \times 10^{18}$ & 290 & 0.23 & 2.3 & \pm 1.1 & 5 & 0.71 & 8.5 \\
(c) & $7.2 \times 10^{18}$ & 256 & 0.23 & 2.7 & \pm 1.8 & 10 & 0.67 & 5.5 \\
(d) & $7.8 \times 10^{18}$ & 227 & 0.26 & 3.9 & \pm 2.8 & 14 & 0.59 & 7.5 \\
(e) & $8.4 \times 10^{18}$ & 156 & 0.31 & 4.1 & \pm 3 & 19 & 0.48 & 19 \\
(f) & $9.6 \times 10^{18}$ & 131 & 0.27 & 4.9 & \pm 3 & 45 & 0.41 & 19 \\
\hline \hline
\end{tabular}
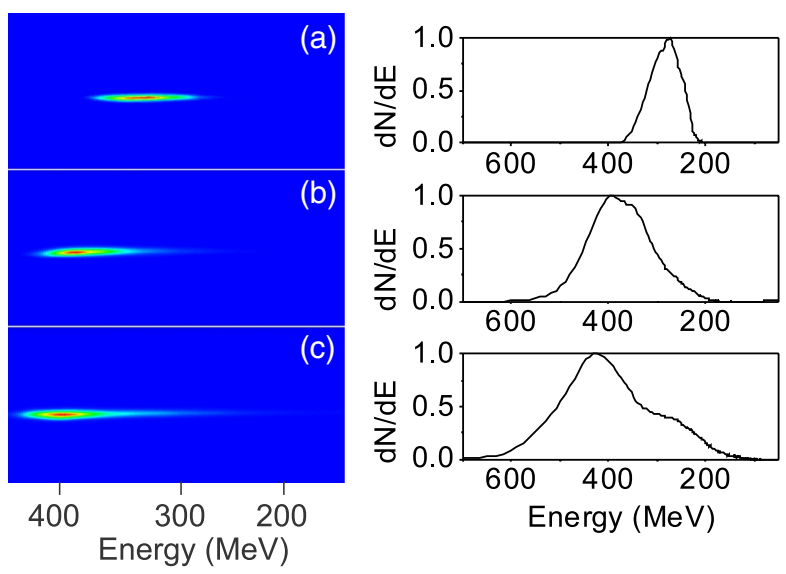

FIG. 9. Images of spectrally dispersed electron beams as a function of laser power and plasma density: (a) $P=40 \mathrm{TW}$, $n_{e}=7.2 \times 10^{18} \mathrm{~cm}^{-3}$; (b) $P=51 \mathrm{TW}, n_{e}=6.4 \times 10^{18} \mathrm{~cm}^{-3}$; (c) $P=58 \mathrm{TW}, n_{e}=5.6 \times 10^{18} \mathrm{~cm}^{-3}$. All images are obtained with a $4 \mathrm{~mm}$ jet and a higher-resolution electron spectrometer. The right column contains electron spectra for each case.

over many campaigns, carried out over an extended period of time, provided the laser and plasma conditions are nominally the same. This stability is important not only for applications, but also has intrinsic value as it allows for systematic study of injection and acceleration processes.

\section{PHYSICAL NATURE OF THE SELF-INJECTION THRESHOLD}

In this section, we concentrate on the self-injection threshold corresponding to the sequence of shots presented in Fig. 4, and propose one possible physical explanation of the observed trend. We explore the dynamics of selfinjection in its relation to the evolution of the driver by means of 3D PIC simulations using the quasicylindrical code CALDER-CIRC [56]. This code exploits axial symmetry, by employing a poloidal mode decomposition for all electromagnetic fields and currents, while retaining full 3D macroparticle dynamics. The near-cylindrical symmetry of interaction permits retaining just the two lowest order poloidal modes. Sampling noise was suppressed by using a large number (45) of macroparticles per cell and high resolution in the direction of propagation, $\Delta z=$ $0.125 \mathrm{c} / \omega_{0}$. The radial grid spacing is $\Delta r=15.6 \Delta z$ and the time step is $\Delta t=0.1244 / \omega_{0}$. For the range of parameters relevant to the reported experiments, simulations with CALDER-CIRC agree quite closely [53] with simulations performed in 3D Cartesian geometry using the PIC code VORPAL [57].

In all simulations, a linearly polarized Gaussian laser pulse with $P=43 \mathrm{TW}$, central wavelength $\lambda_{0}=$ $0.805 \mu \mathrm{m}$, and FWHM duration $\tau_{L}=30 \mathrm{fs}$, was focused into a spot with the radius $r_{0}=13.6 \mu \mathrm{m}$, yielding the Rayleigh length $Z_{R} \approx 0.72 \mathrm{~mm}$. The peak intensity at focus was $1.77 \times 10^{19} \mathrm{~W} / \mathrm{cm}^{2}$, corresponding to a 
normalized vector potential $a_{0}=2.53$. The preionized helium plasma had a trapezoidal profile with $0.5 \mathrm{~mm}$ linear entrance and exit ramps and a $2 \mathrm{~mm}$ central plateau. To allow direct comparison of simulation results with the experimental data, macroparticles exiting the plasma at the end of the simulation were subsequently propagated through the detection system using GPT.

The negligible energy tail [cf. Figs. 4(a)-4(d)] implies that the duration of self-injection is very brief. A physical explanation of this rapid termination of injection is not straightforward. Estimates [55] and simulations [32] indicate that in all cases presented in Fig. 4 beam loading is too weak to suppress self-injection. Additionally, termination of self-injection below the optimal density, $n_{e}<6 \times$ $10^{18} \mathrm{~cm}^{-3}$, cannot be attributed to the insufficient power for self-focusing, self-guiding, and bubble creation as the laser power is still a factor 9 above the threshold for relativistic self-focusing. (The nearly ideal laser pulse ensures efficient coupling of the laser energy to the bubble, in stark contrast to earlier studies $[15,18,19]$.)

The measured electron energy is well below that predicted by the standard scalings [28] (viz. measured $315 \mathrm{MeV}$ against estimated $415 \mathrm{MeV}$ for $n_{e}=$ $\left.6 \times 10^{18} \mathrm{~cm}^{-3}, P=43 \mathrm{TW}\right)$. For beam loading to account for this difference in energy gain, the beam charge would need to be $\sim 50$ times larger [33]. The observed beam energy thus suggests that accelerated electrons exit the plasma long before dephasing. Since the dephasing length is shorter than the length of the density plateau (viz. $L_{\mathrm{deph}} \approx 1.7 \mathrm{~mm}$ for $n_{e}=6 \times 10^{18} \mathrm{~cm}^{-3}$ and $P=$ $43 \mathrm{TW}$ ), injection must have occurred somewhere in the last half of the plasma.

Simulations for the parameters of Figs. 4(a)-4(d), carried out with the laser focus at the foot of the density front ramp, show the laser pulse forming an optical shock long before the end of the plasma, producing a polychromatic tail with nC-scale charge. These simulations predict an order of magnitude more charge in the QME component than seen in the experiments, and also show robust selfinjection below $n_{e}=5 \times 10^{18} \mathrm{~cm}^{-3}$.

In light of the above reasoning, the electron spectra in Figs. 4(a)-4(d) indicate that (a) focusing of the pulse is very slow, delaying formation of the bubble and injection, reducing beam charge, energy, and energy spread; (b) pulse front etching remains insignificant (explaining the negligible background); and (c) as soon as the density drops below a threshold value, the pulse does not focus sufficiently to create a bubble and injection does not occur. All these dynamical features may be attributed to the location of the nonlinear laser focus within the plasma. To explore this, we conducted a series of simulations, varying the positions of the pulse focal plane with respect to the plasma edge. Beams with parameters close to experimental ones [viz. Figs. 4(c) and 4(d)] have been recovered with the laser focus placed $1 \mathrm{~mm}$ (or $1.4 Z_{R}$ ) before the front edge of the plasma. This offset is at the limit of experimental uncertainty of the measured laser focus. With this focal location, simulations also confirm that, at densities below $5.5 \times$ $10^{18} \mathrm{~cm}^{-3}$, a bubble does not form and self-injection into the first bucket does not occur.

Simulated and measured energy spectra are presented in Figs. 10(a) and 10(b) for the parameters of Figs. 4(c) and 4(d), respectively. The simulation with plasma parameters of Fig. 4(c) [spectra in Fig. 10(a), $P=43 \mathrm{TW}, n_{e}=7.2 \times$ $10^{18} \mathrm{~cm}^{-3}$ ] shows that locating the focal spot before the jet significantly reduces the low-energy background in the beam. With this focal location, the pulse is diverging when it enters the plasma, taking nearly $2 / 3$ of the total plasma length to focus sufficiently to create a bubble and initiate injection. When the bubble forms this close to the end of the plasma, pulse front etching is insignificant and continuous injection occurs at a low level, contributing to the weak tail below $200 \mathrm{MeV}$. In addition, electrons produced by continuous injection are poorly collimated, further reducing their signal after magnetic dispersion in the detector. Details of the plasma wake evolution and the dynamics of electron self-injection in this case are discussed in detail in Ref. [16] (cf. Fig. 5 of that paper). At higher plasma density, $n_{e}=7.8 \times 10^{18} \mathrm{~cm}^{-3}$, the pulse focuses more rapidly and injection begins earlier, resulting in higher injected charge and a broader energy spectrum as shown in Fig. 10(b). Energy of the electron beams obtained in the simulations is close to the experimentally measured values [entries (c) and (d) of Table I]. At the same time, the simulations overestimate the beam charge and divergence roughly by a factor of 3 . Importantly, in both cases, the beam electrons are far from dephasing; delayed self-injection occurs at less than a millimeter from the end of the plasma plateau. Given the cold wave
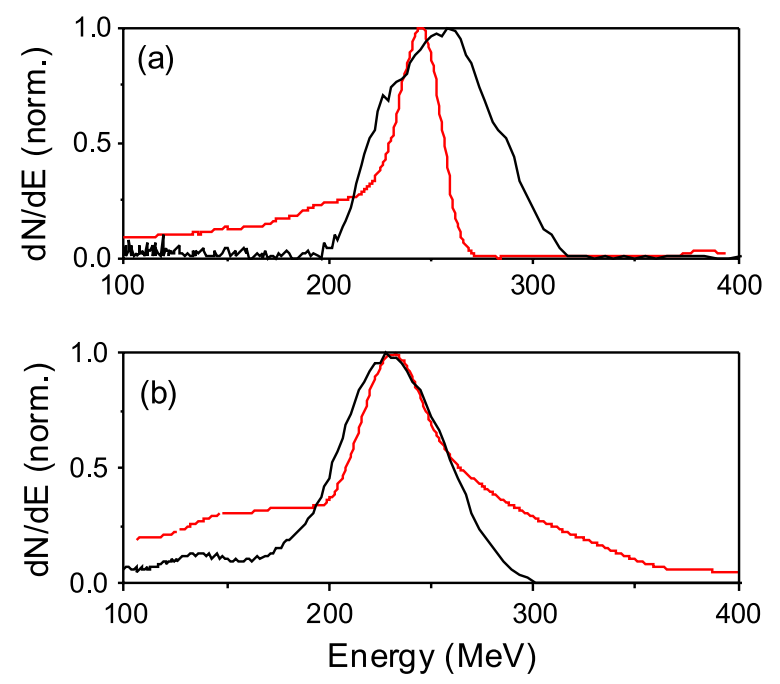

FIG. 10. Lineouts of experimental (black), and simulated (red) electron energy spectra for the parameters (a) of Fig. 4(c) $\left(P=43 \mathrm{TW}, n_{e}=7.2 \times 10^{18} \mathrm{~cm}^{-3}\right)$ and (b) of Fig. 4(d) $\left(P=43 \mathrm{TW}, n_{e}=7.8 \times 10^{18} \mathrm{~cm}^{-3}\right)$. 
breaking electric field $E_{W B} \sim 260 \mathrm{MV} / \mathrm{mm}$ (where $\left.E_{W B}=0.96 \sqrt{n_{e}\left[\mathrm{~cm}^{-3}\right]} \mathrm{V} / \mathrm{cm}\right)$, and knowing that accelerating gradient inside the bubble actually exceeds this value, we find the simulation results self-consistent.

\section{CONCLUSIONS}

This paper reports the results of the laser-plasma acceleration experiments carried out in the near-threshold regime for electron self-injection. For the first time, stable operation in this regime is demonstrated. Sensitivity of the near-threshold regime to the fluctuations of laser and plasma parameters are mitigated by the nearly Gaussian laser pulse and stable gas-jet target, minimizing the effect of plasma nonlinearities on electron-beam characteristics. The results of 3D PIC simulations indicate that production of the optimal beams in the laboratory may be associated with the precise location of the laser pulse focus and transient dynamics of the laser pulse in plasma, rather than with the stable self-guiding of the pulse until electron dephasing and/or pulse depletion. The hallmark of the near-threshold regime, the QME high-energy component remains the dominant feature of measured electron spectra, and shows minimal fluctuations of parameters over long series of shots. Furthermore, 250-430 MeV electron beams with no detectable low-energy background are produced in $50 \%-100 \%$ of shots at reduced plasma density, $<7.2 \times$ $10^{18} \mathrm{~cm}^{-3}$, and 40-60 TW laser power. The quality of these monoenergetic beams remains high over a broad range of laser and plasma parameters. This stable and scalable operation of laser-plasma accelerator is an asset for radiation-physics applications [39] and is an important scientific instrument for studies of the fundamental processes underlying the acceleration process-self-injection of electrons and its relation to the nonlinear optical processes.

\section{ACKNOWLEDGMENTS}

The work of S. B., S. Y. K., N. D. P., G. G., V. R., N. J. C., K. J. B., S. C., I. G., B. A. S., and D. P. U. was in part supported by the U.S. DOE Grant No. DE-FG02-05ER15663; DARPA Grant No. FA9550-09-1-0009; DTRA Grant No. HDTRA1-11-C-0001; AFOSR Grant No. FA955011-1-0157; and the U.S. DHS Grant No. 2007-DN-077ER0007-02. The DIOCLES laser is supported by the AFOSR under Contracts No. FA 9550-08-1-0232 and No. FA9550-07-1-0521. The work of S. Y. K. and B. A. S. was also in part supported by U.S. DOE Grants No. DEFG02-08ER55000 and No. DE-SC0008382. The work of B. M. C. and D. L. B. was partially supported by U.S. DOE Contracts No. DE-SC0006245 (SBIR) and No. DEFC0207ER41499 (SciDAC). VORPAL simulations used resources of the National Energy Research Scientific Computing Center, which is supported by the Office of Science of the U.S. DOE under Contract No. DE-AC02-05CH11231.
A. B. and E. L. were supported by LASERLAB-EUROPE/ LAPTECH through EC FP7 Contract No. 228334. The authors thank Centre de Calcul Recherche et Technologie at Bruyères-le-Chàtel (http://www-ccrt.cea.fr) for providing high-performance computing resources. We also thank Dr. Sicong Li of the UNL Medical Center for providing access to the $18 \mathrm{MeV}$ accelerator.

[1] S.P.D. Mangles, C. D. Murphy, Z. Najmudin, A. G. R. Thomas, J. L. Collier, A.E. Dangor, E. J. Divall, P. S. Foster, J. G. Gallacher, C. J. Hooker, D. A. Jaroszynski, A. J. Langley, W. B. Mori, P. A. Norreys, F. S. Tsung, R. Viskup, B. R. Walton, and K. Krushelnick, Nature (London) 431, 535 (2004).

[2] C. G. D. Geddes, Cs. Toth, J. van Tilborg, E. Esarey, C. B. Schroeder, D. Bruhwiler, C. Nieter, J. Cary, and W.P. Leemans, Nature (London) 431, 538 (2004).

[3] J. Faure, Y. Glinec, A. Pukhov, S. Kiselev, S. Gordienko, E. Lefebvre, J.-P. Rousseau, F. Burgy, and V. Malka, Nature (London) 431, 541 (2004).

[4] V. Malka, J. Faure, Y. Glinec, A. Pukhov, and J.-P. Rousseau, Phys. Plasmas 12, 056702 (2005).

[5] S.P.D. Mangles, A. G. R. Thomas, M.C. Kaluza, O. Lundh, F. Lindau, A. Persson, F. S. Tsung, Z. Najmudin, W. B. Mori, C.-G. Wahlström, and K. Krushelnick, Phys. Rev. Lett. 96, 215001 (2006).

[6] S. P. D. Mangles, A. G. R. Thomas, O. Lundh, F. Lindau, M. C. Kaluza, A. Persson, C.-G. Wahlström, K. Krushelnick, and Z. Najmudin, Phys. Plasmas 14, 056702 (2007).

[7] J. Osterhoff, A. Popp, Zs. Major, B. Marx, T. P. RowlandsRees, M. Fuchs, M. Geissler, R. Hörlein, B. Hidding, S. Becker, E. A. Peralta, U. Schramm, F. Grüner, D. Habs, F. Krausz, S. M. Hooker, and S. Karsch, Phys. Rev. Lett. 101, 085002 (2008).

[8] A. Maksimchuk, S. Reed, N. Naumova, V. Chvykov, B. Hou, G. Kalintchenko, T. Matsuoka, J. Nees, P. Rousseau, G. Mourou, and V. Yanovsky, Appl. Phys. B 89, 201 (2007).

[9] K. Schmid, L. Veisz, F. Tavella, S. Benavides, R. Tautz, D. Herrmann, A. Buck, B. Hidding, A. Marcinkevicius, U. Schramm, M. Geissler, J. Meyer-ter-Vehn, D. Habs, and F. Krausz, Phys. Rev. Lett. 102, 124801 (2009).

[10] K. Schmid, A. Buck, C. M. S. Sears, J. M. Mikhailova, R. Tautz, D. Herrmann, M. Geissler, F. Krausz, and L. Veisz, Phys. Rev. ST Accel. Beams 13, 091301 (2010).

[11] R. C. Issac, G. Vieux, G. H. Welsh, R. Shanks, E. Brunetti, S. Cipiccia, M.-P. Anania, X. Yang, S. M. Wiggins, M. R. Islam, B. Ersfeld, J. Farmer, G. Raj, S. Chen, D. Clark, T. McCanny, and D.A. Jaroszynski, in Harnessing Relativistic Plasma Waves as Novel Radiation Sources from Terahertz to X-Rays and Beyond, edited by D. A. Jaroszynski and A. Rousse, Proc. SPIE Int. Soc. Opt. Eng. Vol. 7359 (SPIE, Bellingham, WA, 2009), p. 735915.

[12] S. M. Wiggins, R. C. Issac, G. H. Welsh, E. Brunetti, R. P. Shanks, M.P. Anania, S. Cipiccia, G. G. Manahan, C. Aniculaesei, B. Ersfeld, M. R. Islam, R. T. L. Burgess, G. Vieux, W. A. Gillespie, A. M. MacLeod, S. B. van der 
Geer, M. J. de Loos, and D. A. Jaroszynski, Plasma Phys. Controlled Fusion 52, 124032 (2010).

[13] A. J. Gonsalves, K. Nakamura, C. Lin, D. Panasenko, S. Shiraishi, T. Sokollik, C. Benedetti, C. B. Schroeder, C. G. R. Geddes, J. van Tilborg, J. Osterhoff, E. Esarey, C. Toth, and W. P. Leemans, Nat. Phys. 7, 862 (2011).

[14] N. A. M. Hafz, T. M. Jeong, I. Choi, S. K. Lee, K. H. Pae, V. V. Kulagin, J.H. Sung, T. J. Yu, K.-H. Hong, T. Hosokai, J. R. Cary, D.-K. Ko, and J. Lee, Nat. Photonics 2, 571 (2008).

[15] S. Kneip et al., Plasma Phys. Controlled Fusion 53, 014008 (2011).

[16] S. Banerjee, N.D. Powers, V. Ramanathan, I. Ghebregziabher, K. J. Brown, C. M. Maharjan, S. Chen, A. Beck, E. Lefebvre, S. Y. Kalmykov, B. A. Shadwick, and D.P. Umstadter, Phys. Plasmas 19, 056703 (2012).

[17] W. P. Leemans, B. Nagler, A. J. Gonsalves, Cs. Tóth, K. Nakamura, C. G. R. Geddes, E. Esarey, C. B. Schroeder, and S. M. Hooker, Nat. Phys. 2, 696 (2006).

[18] S. Kneip, S. R. Nagel, S. F. Martins, S. P. D. Mangles, C. Bellei, O. Chekhlov, R. J. Clarke, N. Delerue, E. J. Divall, G. Doucas, K. Ertel, F. Fiuza, R. Fonseca, P. Foster, S. J. Hawkes, C. J. Hooker, K. Krushelnick, W. B. Mori, C. A. J. Palmer, K. Ta Phuoc, P. P. Rajeev, J. Schreiber, M. J. V. Streeter, D. Urner, J. Vieira, L. O. Silva, and Z. Najmudin, Phys. Rev. Lett. 103, 035002 (2009).

[19] D. Froula et al., Phys. Rev. Lett. 103, 215006 (2009).

[20] J.E. Ralph, C. E. Clayton, F. Albert, B. B. Pollock, S. F. Martins, A. E. Pak, K. A. Marsh, J. L. Shaw, A. Till, J. P. Palastro, W. Lu, S. H. Glenzer, L. O. Silva, W. B. Mori, C. Joshi, and D. H. Froula, Phys. Plasmas 17, 056709 (2010).

[21] X. Wang et al., in Advanced Accelerator Concepts: 15th Workshop, edited by R. Zgadzaj, E. Gaul, and M.C. Downer, AIP Conf. Proc. No. 1507 (American Institute of Physics, New York, 2012), pp. 341-344.

[22] P. Dong, S. A. Reed, S. A. Yi, S. Kalmykov, G. Shvets, M. C. Downer, N. H. Matlis, W. P. Leemans, C. McGuffey, S.S. Bulanov, V. Chvykov, G. Kalintchenko, K. Krushelnick, A. Maksimchuk, T. Matsuoka, A. G. R. Thomas, and V. Yanovsky, Phys. Rev. Lett. 104, 134801 (2010).

[23] P. Dong, S. A. Reed, S. A. Yi, S. Kalmykov, Z. Y. Li, G. Shvets, N. H. Matlis, C. McGuffey, S.S. Bulanov, V. Chvykov, G. Kalintchenko, K. Krushelnick, A. Maksimchuk, T. Matsuoka, A.G. R. Thomas, V. Yanovsky, and M. C. Downer, New J. Phys. 12, 045016 (2010).

[24] M. H. Helle, D. Kaganovich, D. F. Gordon, and A. Ting, Phys. Rev. Lett. 105, 105001 (2010).

[25] J. B. Rosenzweig, B. Breizman, T. Katsouleas, and J. J. Su, Phys. Rev. A 44, R6189 (1991).

[26] P. Mora and T. M. Antonsen, Jr., Phys. Rev. E 53, R2068 (1996).

[27] A. Pukhov and J. Meyer-ter-Vehn, Appl. Phys. B 74, 355 (2002).

[28] W. Lu, M. Tzoufras, C. Joshi, F. S. Tsung, W. B. Mori, J. Vieira, R. A. Fonseca, and L. O. Silva, Phys. Rev. ST Accel. Beams 10, 061301 (2007).

[29] S. Y. Kalmykov et al., New J. Phys. 12, 045019 (2010).
[30] S. Y. Kalmykov, A. Beck, S. A. Yi, V. Khudik, B. A. Shadwick, E. Lefebvre, and M. C. Downer, in Advanced Accelerator Concepts: 14th Workshop, edited by S.H. Gold and G. S. Nusinovich, AIP Conf. Proc. No. 1299 (American Institute of Physics, New York, 2010), pp. 174-179.

[31] S. Y. Kalmykov, S. A. Yi, A. Beck, A. F. Lifschitz, X. Davoine, E. Lefebvre, V. Khudik, G. Shvets, and M.C. Downer, Plasma Phys. Controlled Fusion 53, 014006 (2011).

[32] S. Y. Kalmykov, A. Beck, S. A. Yi, V. N. Khudik, M. C. Downer, E. Lefebvre, B. A. Shadwick, and D.P. Umstadter, Phys. Plasmas 18, 056704 (2011).

[33] S. Y. Kalmykov, A. Beck, X. Davoine, E. Lefebvre, and B. A. Shadwick, New J. Phys. 14, 033025 (2012).

[34] V. Ramanathan, S. Banerjee, N. Powers, N. Cunningham, N. A. Chandler-Smith, Kun Zhao, K. Brown, D. Umstadter, S. Clarke, S. Pozzi, J. Beene, C. R. Vane, and D. Schultz, Phys. Rev. ST Accel. Beams 13, 104701 (2010).

[35] S. Kneip et al., Nat. Phys. 6, 980 (2010).

[36] S. Kneip, C. McGuffey, F. Dollar, M.S. Bloom, V. Chvykov, G. Kalintchenko, K. Krushelnick, A. Maksimchuk, S.P.D. Mangles, T. Matsuoka, Z. Najmudin, C. A. J. Palmer, J. Schreiber, W. Schumaker, A. G. R. Thomas, and V. Yanovsky, Appl. Phys. Lett. 99, 093701 (2011).

[37] S. Fourmaux, S. Corde, K. Ta Phuoc, P. Lassonde, G. Lebrun, S. Payeur, F. Martin, S. Sebban, V. Malka, A. Rousse, and J. C. Kieffer, Opt. Lett. 36, 2426 (2011).

[38] S. Cipiccia et al., Nat. Phys. 7, 867 (2011).

[39] F. V. Hartemann, D. J. Gibson, W. J. Brown, A. Rousse, K. Ta Phuoc, V. Malka, J. Faure, and A. Pukhov, Phys. Rev. ST Accel. Beams 10, 011301 (2007).

[40] V. Malka, Phys. Plasmas 19, 055501 (2012), and references therein.

[41] M. Wen, B. Shen, X. Zhang, F. Wang, Zh. Jin, L. Ji, W. Wang, J. Xu, and K. Nakajima, New J. Phys. 12, 04510 (2010).

[42] M.S. Hur and H. Suk, Phys. Plasmas 18, 033102 (2011).

[43] A. K. Upadhyay, S. A. Samant, D. Sarkar, P. Jha, and S. Krishnagopal, Phys. Plasmas 18, 033109 (2011).

[44] F.S. Tsung, C. Ren, L. O. Silva, W. B. Mori, and T. Katsouleas, Proc. Natl. Acad. Sci. U.S.A. 99, 29 (2002).

[45] J. Faure, Y. Glinec, J. J. Santos, F. Ewald, J. P. Rousseau, S. Kiselev, A. Pukhov, T. Hosokai, and V. Malka, Phys. Rev. Lett. 95, 205003 (2005).

[46] General Particle Tracer (GPT) code, Pulsar Physics, The Netherlands [http://www.pulsar.nl/gpt].

[47] S. B. van der Geer, O. J. Luiten, M. J. de Loos, G. Pöplau, and U. van Rienen, in Computational Accelerator Physics 2002, edited by M. Berz and K. Makino, Institute of Physics Conference Series 175 (IoP Publishing, Bristol, UK, 2005), pp. 101-110.

[48] S.P.D. Mangles, A. G. R. Thomas, M.C. Kaluza, O. Lundh, F. Lindau, A. Persson, Z. Najmudin, C.-G. Wahlström, C.D. Murphy, C. Kamperidis, K. L. Lancaster, E. Divall, and K. Krushelnick, Plasma Phys. Controlled Fusion 48, B83 (2006). 
[49] B. Hidding, M. Geissler, G. Pretzler, K.-U. Amthor, H. Schwoerer, S. Karsch, L. Veisz, K. Schmid, and R. Sauerbrey, Phys. Plasmas 16, 043105 (2009).

[50] G.-Z. Sun, E. Ott, Y. C. Lee, and P. Guzdar, Phys. Fluids 30, 526 (1987).

[51] K. Németh, B. Shen, Y. Li, H. Shang, R. Crowell, K. C. Harkay, and J.R. Cary, Phys. Rev. Lett. 100, 095002 (2008).

[52] J. Vieira, F. Fiúza, L. O. Silva, M. Tzoufras, and W. B. Mori, New J. Phys. 12, 045025 (2010).

[53] B. M. Cowan, S. Y. Kalmykov, A. Beck, X. Davoine, K. Bunkers, A.F. Lifschitz, E. Lefebvre, D. L. Bruhwiler,
B. A. Shadwick, and D. P. Umstadter, J. Plasma Phys. 78, 469 (2012).

[54] F. S. Tsung, R. Narang, W. B. Mori, C. Joshi, R. A. Fonseca, and L. O. Silva, Phys. Rev. Lett. 93, 185002 (2004).

[55] M. Tzoufras, W. Lu, F. S. Tsung, C. Huang, W. B. Mori, T. Katsouleas, J. Vieira, R. A. Fonseca, and L. O. Silva, Phys. Plasmas 16, 056705 (2009).

[56] A. F. Lifschitz, X. Davoine, E. Lefebvre, J. Faure, C. Rechatin, and V. Malka, J. Comput. Phys. 228, 1803 (2009).

[57] C. Nieter and J. R. Cary, J. Comput. Phys. 196, 448 (2004). 